\title{
An iterative compositional model for subsurface multiphase flow
}

\author{
Howard W. Reeves ${ }^{\mathrm{a}}$ and Linda M. Abriola ${ }^{\mathrm{b}}$ \\ ${ }^{a}$ Department of Geological Sciences, University of South Carolina, Columbia, SC 29208, USA \\ ${ }^{b}$ Department of Civil and Environmental Engineering, The University of Michigan, Ann Arbor, \\ MI 48109-2125, USA
}

(Received October 6, 1992; revised and accepted December 1, 1993)

\begin{abstract}
The governing equations describing multiphase flow with multicomponent solute transport may be cast in a number of forms. While mathematically equivalent, the numerical models based on each formulation differ in flexibility and efficiency. The set-iterative compositional formulation, a mathematical formulation that separates the solution of the phase-balance equations from the species balance is described herein. A mathematical model based on this formulation describes the flow of two mobile phases. Each phase may transport multiple chemical components. Mass exchange between phases is expressed by a linear kinetic equation. The numerical model based on the set-iterative compositional formulation is shown to model non-equilibrium phase partitioning, to provide a flexible framework that may be applied to organic mixtures with differing numbers of components and to yield a more efficient solution in comparison with models arising from standard compositional formulations.
\end{abstract}

\section{INTRODUCTION}

Remediation of groundwater systems contaminated by organic chemicals has become a national priority due to the potentially adverse health effects of these pollutants and to their widespread use. Tools employed in the study of the movement and fate of non-aqueous-phase organic liquids include mathematical models. Mathematical simulations allow researchers to test hypotheses, and may lead to a greater understanding of the phenomena governing multiphase flow and transport. Often the mathematical equations describing flow of phases and transport of components within a phase must be solved numerically. In this case, the mathematical formulation of the governing equations greatly influences the efficiency and accuracy of the final numerical model.

A number of numerical models have been developed to simulate multiphase 
flow with mass transport in the subsurface. The objectives of this paper are to introduce a numerical model that addresses problems identified with previous modeling efforts, to examine the mathematical formulation of the numerical model and to discuss the performance of the model for several test problems. The presented numerical model is based upon a computationally decoupled formulation of the governing equations and is referred to herein as the setiterative compositional formulation. Other formulations of the governing equations, which have appeared in the literature, are described and compared with the set-iterative compositional model in terms of model flexibility, ease of implementation and efficiency.

\section{BACKGROUND}

A rigorous mathematical description of multiphase flow in the subsurface is based upon mass-balance equations for each component present in the system. The mass-balance equation for a species $i$ in a phase $\alpha$ may be written as (Hassanizadeh and Gray, 1979):

$\frac{\partial}{\partial t}\left(\rho^{\alpha} \epsilon_{\alpha} \omega_{i}^{\alpha}\right)+\nabla \cdot\left(\rho^{\alpha} \epsilon_{\alpha} \omega_{i}^{\alpha} v^{\alpha}\right)+\nabla \cdot \boldsymbol{J}_{i}^{\alpha}=E_{i}^{\alpha}+\omega_{i}^{\alpha} \sigma^{\alpha}$

where $i=$ species $\left(i=1, \ldots, N_{\mathrm{s}}\right.$; with $N_{\mathrm{s}}=$ number of species in the $\alpha$ phase); $\rho^{\alpha}=$ density of the $\alpha$ phase [ $\mathrm{M} \mathrm{L}^{-3}$ ]; $\epsilon_{\alpha}=$ volume fraction of the $\alpha$ phase (dimensionless); $\omega_{i}^{\alpha}=$ mass fraction of species $i$ in the $\alpha$ phase (dimensionless); $\boldsymbol{v}^{\alpha}=$ velocity of the $\alpha$ phase $\left[\mathrm{L} \mathrm{T}^{-1}\right] ; \boldsymbol{J}_{i}^{\alpha}=$ non-advective flux of species $i$ in the $\alpha$ phase $\left[\mathrm{M} \mathrm{T}^{-1} \mathrm{~L}^{-2}\right] ; E_{i}^{\alpha}=$ source term arising from mass exchange between phases $\left[\mathrm{M} \mathrm{T}^{-1} \mathrm{~L}^{-3}\right]$; and $\sigma^{\alpha}=$ external source of phase $\alpha\left[\mathrm{M} \mathrm{T}^{-1}\right.$ $\left.\mathrm{L}^{-3}\right]$.

The first term in this expression represents the accumulation of component $i$ in the $\alpha$ phase. Advective transport of $i$ is described by the second term. The third term accounts for the transport of $i$ in the $\alpha$ phase due to non-advective mechanisms. The right-hand side of Eq. 1 accounts for component mass change in the $\alpha$ phase due to mass exchange with the other phases present in the system and the gain or loss of mass in the $\alpha$ phase due to external sources and sinks.

Darcy's law, modified to account for multiphase flow, is generally used to express the phase velocity as a function of phase pressure and elevation head (Bear, 1972):

$\boldsymbol{v}^{\alpha}=-\left[\frac{\boldsymbol{k} k_{\mathrm{r} \alpha}}{\epsilon_{\alpha} \mu^{\alpha}}\left(\nabla P^{\alpha}-\rho^{\alpha} \boldsymbol{g}\right)\right]$

In this equation, $\boldsymbol{k}=$ intrinsic permeability tensor of the porous medium $\left[\mathrm{L}^{2}\right]$; $k_{\mathrm{r} \alpha}=$ relative permeability of the $\alpha$ phase (dimensionless); $\mu^{\alpha}=$ dynamic 
viscosity of the $\alpha$ phase $\left[\mathrm{M} \mathrm{L}^{-1} \mathrm{~T}^{-1}\right] ; P^{\alpha}=$ pressure of the $\alpha$ phase $\left[\mathrm{M} \mathrm{L}^{-1}\right.$ $\left.\mathrm{T}^{-2}\right]$; and $g=$ gravity acceleration vector $\left[\mathrm{L} \mathrm{T}^{-2}\right]$.

The non-advective flux of a species in a given phase is represented by a Fickian form (Bear, 1972):

$\boldsymbol{J}_{i}^{\alpha}=-\rho^{\alpha} \epsilon_{\alpha} \boldsymbol{D}_{i}^{\alpha} \nabla \omega_{i}^{\alpha}$

For a one-dimensional domain, $\boldsymbol{D}_{i}^{\alpha}$ may be expressed as:

$\boldsymbol{D}_{i}^{\alpha}=D^{\mathrm{m} \alpha^{e}}+a^{\alpha}\left|\boldsymbol{v}^{\alpha}\right|$

while in two or three dimensions, for an isotropic medium, $\boldsymbol{D}_{i}^{\alpha}$ becomes:

$\boldsymbol{D}_{i}^{\alpha}=D^{\mathrm{m} \alpha^{e}}+a_{\mathrm{t}}^{\alpha}\left|\boldsymbol{v}^{\alpha}\right| \delta_{m n}+\left(a_{1}^{\alpha}-a_{\mathrm{t}}^{\alpha}\right) \frac{v_{m}^{\alpha} v_{n}^{\alpha}}{\left|\boldsymbol{v}^{\alpha}\right|}$

where $D^{\mathrm{m} \alpha^{\mathrm{e}}}=$ effective molecular diffusion coefficient $\left[\begin{array}{lll}\mathrm{L}^{2} & \mathrm{~T}^{-1}\end{array}\right] ; a^{\alpha}=$ dispersivity of the $\alpha$ phase [L]; $a_{1}^{\alpha}=$ longitudinal dispersivity of the $\alpha$ phase $[\mathrm{L}] ; a_{\mathrm{t}}^{\alpha}=$ transverse dispersivity of the $\alpha$ phase [L]; $\delta_{m n}=$ Kronecker delta; $v_{\mathrm{m}}^{\alpha}=$ magnitude of velocity of the $\alpha$ phase in the $m$ direction $\left[\mathrm{L} \mathrm{T}^{-1}\right]$; and $\left|v^{\alpha}\right|=$ magnitude of the velocity of the $\alpha$ phase $\left[\mathrm{L} \mathrm{T}^{-1}\right]$.

An important constitutive relationship that is used to close the equation set for all multiphase flow models is the relationship between capillary pressure, which is defined as the pressure difference between two contiguous fluids, and fluid saturation. Although this relationship may exhibit hysteresis, for simplicity, the formulations described in this paper use a single-valued capillary pressure-saturation function. A constitutive expression is also required to describe relative permeability. A number of models have been developed that predict the relationship between saturation and relative permeability based upon capillary pressure-saturation information (Mualem, 1976; van Genuchten, 1980; Parker et al., 1987b). The particular forms used for these constitutive relationships in the simulations described herein are summarized in Appendices $\mathrm{A}-\mathrm{C}$.

Another important aspect of multiphase, multicomponent transport models is the exchange of mass between phases. This mass exchange is generally assumed to have no effect on the physical properties of the water phase. An organic or gas phase, however, is usually modeled with properties dependent on phase composition. The correlations used to estimate phase properties from composition are described by Abriola and Pinder (1985a), and Kaluarachchi and Parker (1990). The actual mass exchange may be modeled by assuming local equilibrium or by employing a mass-transfer expression. If local equilibrium between the phases is assumed the following relationships hold:

$\omega_{i}^{\alpha}=K_{i}^{\alpha \beta} \omega_{i}^{\beta}$ 
where $K_{i}^{\alpha \beta}=$ equilibrium coefficient between the $\alpha$ and $\beta$ phases (dimensionless).

The equilibrium coefficients may be calculated using solubility and vapor pressure data.

Kinetically controlled mass exchange between phases is often described by a simple linear driving force expression in the form (Treybal, 1980):

$E_{i}^{\alpha}=\bar{k}_{i}^{\alpha \beta}\left(\omega_{i}^{\alpha^{*}}-\omega_{i}^{\alpha}\right)$

where $\omega_{i}^{\alpha^{*}}=$ mass fraction of species $i$ in the $\alpha$ phase that is in equilibrium with the mass fraction of $i$ in the $\beta$ phase; and $\bar{k}_{i}^{\alpha \beta}=$ lumped mass-exchange coefficient $\left[\mathrm{M} \mathrm{T}^{-1} \mathrm{~L}^{-3}\right]$.

The lumped mass-exchange coefficient is a function of the fluids, the velocity of the fluids, the exchanging species and the interfacial area available for exchange. Both the available interfacial area and the fluid velocities are strong functions of the porous-medium characteristics. The potential importance of rate-limited mass exchange between entrapped non-aqueous-phase liquids and water in the subsurface has been explored by many researchers (see, e.g., Powers et al., 1991, 1992; Brusseau, 1992).

In addition to these basic governing equations, several constraints are imposed on the system by definition:

$\sum_{i=1}^{N_{\mathrm{s}}} \omega_{i}^{\alpha}$

$s_{\mathrm{o}}+s_{\mathrm{w}}+s_{\mathrm{g}}=1$

where $s_{\mathrm{o}}, s_{\mathrm{W}}, s_{\mathrm{g}}=$ fluid saturation of the organic, water and gas phase, respectively.

The total mass gained or lost by a phase due to mass exchange with other phases may be expressed as:

$\sum_{i=1}^{N_{\mathrm{s}}} E_{i}^{\alpha}=E^{\alpha}$

If the species are not transformed by chemical reaction or biodegradation, each species is conserved over the system:

$\sum_{\alpha=1}^{N_{\mathrm{p}}} E_{i}^{\alpha}=0$

where $N_{\mathrm{p}}=$ the number of phases present.

The governing equations (Eqs. 1-11) describing multiphase flow with multicomponent transport are coupled non-linear partial differential 
equations that do not admit general analytical solution. The efficiency and accuracy of numerical approximations to these governing equations are strongly influenced by the selected formulation. Three formulations that have been presented in the literature are reviewed below. All formulations include constitutive relationships, partitioning relationships, mass exchange, and Darcy's and Fick's laws as described above. For each formulation any appropriate numerical technique may be applied to approximate the governing equations. Each formulation, however, results in a different iterative procedure for solution of the discretized equations.

\section{Simultaneous formulation}

In a simultaneous formulation, governing equations relating the capillary pressure between the fluids, the fluid saturations, and the mass fraction of components within each phase are described through a single set of equations. Within the equation set, the capillary pressure and mass fractions are used as the primary variables. Fluid saturation is expressed as a function of capillary pressure through a constitutive relationship. Thus, saturation, capillary pressure and mass fraction are approximated simultaneously. The simultaneous formulation may be the most computationally burdensome of the formulations discussed since all variables are approximated in a single step. Examples of the simultaneous solution method may be found in Abriola and Pinder (1985a, b), and Sleep and Sykes (1990). Abriola (1984) used this type of formulation to describe the motion of two mobile phases in the subsurface. A two-component organic phase is modeled. One component is allowed to partition and be transported in the water and (static) air phases. Three governing partial differential equations were used to describe the system: a water-phase mass balance, a species mass balance on the inert organic component and a species mass balance on the volatile organic component. Results from this model will be used in the following sections as representative of the simultaneous approach.

\section{Adaptive IMPES formulation}

One modification to the simultaneous formulation arises when the coupling between the fluid saturations and capillary pressures is lagged by one solution step. In the implicit pressure-explicit saturation (IMPES) formulation, the general balance equations are combined to eliminate the saturation unknowns via Eq. 10. The assumption that the capillary pressure between the phases remains constant over a time step allows the equations to be written in terms of one unknown phase pressure. After this pressure is determined, one of the phase mass-balance equations may then be used to explicitly 
determine the phase saturation. Once the new phase saturation is determined, the capillary pressure between the phases is updated through the use of the appropriate constitutive relationship. While this formulation yields a small matrix problem for the pressure and an efficient explicit determination of the secondary unknowns, its efficiency may be limited by convergence behavior that restricts the model to small time steps (Forsyth and Sammon, 1986).

To take advantage of the efficiency of the IMPES formulation while retaining the stability of the simultaneous formulation, Forsyth and Sammon (1986) propose an adaptive implicit procedure. The governing equations are solved in a simultaneous fashion in regions of the domain where the IMPES form would be unstable. The efficient IMPES method is used in the remainder of the domain. The method is developed for a three-phase "black-oil" simulator (Forsyth and Sammon, 1986), and is extended by Forsyth (1988) to a twomobile-phase compositional model. This formulation only considers a singlecomponent organic phase, but the computational advantages offered by this approach warrant its description herein. The basic governing equations resemble those derived by Abriola (1984). The criteria used to determine if a block may be considered an IMPES block and the manner in which the Jacobian matrix is evaluated are described by Forsyth and Sammon (1986), and the impact of mass exchange on stability is evaluated by Forsyth (1988). Forsyth (1988) determined that once mass is allowed to exchange between phases, most of the cell blocks require simultaneous solution. In addition to the adaptive implicit approach, a variable substitution technique is implemented into the numerical model to allow for a zero saturation of the organic phase (Forsyth, 1988). The variable substitution circumvents the requirement of the simultaneous formulations that the organic phase must exist throughout the domain and prevents smearing of the organic-phase saturation front arising from the introduction of an artificial amount of organic in the domain.

One disadvantage of the formulations presented above is that the number of species comprising the organic phase in each formulation is fixed. While these formulations may be extended to consider multicomponent mixtures, such a procedure might not produce a practical model. The equation set resulting from a simultaneous formulation becomes quite large, and the resulting numerical model will be computationally expensive. The adaptive IMPES formulation works well for a single-component organic, but as more components are introduced to the problem, additional mass-balance equations must be written for each component. Thus, the total number of unknowns increases and the efficiency resulting from the IMPES procedure is reduced. Again the equation set becomes large, leading to a computationally expensive model. Additionally, for many interacting components, general algorithms for the variable substitution procedure may be difficult to formulate. 


\section{Computationally decoupled formulation}

An alternative formulation may be developed if equations describing the movement of the bulk phases are separated from those describing the transport of components within the phases. This formulation produces two sets of non-linear partial differential equations. These sets are weakly coupled by compositionally dependent fluid properties and by mass-exchange terms. In general, an iterative scheme is required to account for this coupling between equation sets. This type of formulation may be called a computationally decoupled formulation (Kaluarachchi and Parker, 1990). In the hydrology literature, models based on a computationally decoupled formulation were proposed by Falta and Javendel (1987), Parker et al. (1987a), and Reeves and Abriola (1988). Kaluarachchi and Parker (1990), and Katyal et al. (1991) presented modified versions of the model proposed earlier by Parker et al. (1987a). Ryan and Cohen (1991) also used a computationally decoupled formulation for a one-dimensional front-tracking algorithm. The formulation presented in this paper may also be classified as a computationally decoupled formulation.

In general, a computationally decoupled formulation offers advantages in terms of model flexibility, as any number of components may be studied without changing the solution of the phase-balance equations or the solution approach for the species-balance equations. For equivalent problems, a computationally decoupled formulation is expected to be more efficient than a simultaneous formulation due to the solution of smaller matrix problems. Unfortunately, the most straightforward iterative scheme proposed for a computationally decoupled model introduces error into the solution of the equations. The sources of these errors are described in more detail in the following section. Two remedies for the numerical difficulties may be proposed: modification of the iterative solution technique (Kaluarachchi and Parker, 1990) and reformulation of the species-balance equations. This second approach is the foundation for the set-iterative model, and is developed in detail below.

\section{MODEL FORMULATION}

The set-iterative compositional model presented in this paper is a computationally decoupled approach based on two-sets of non-linear partial differential equations subject to appropriate initial and boundary conditions. The first set of equations describes the phase mass balance of two mobile phases in the subsurface, and is obtained by summing the general mass-balance equation (1) over the species present. Due to the mass fraction constraint (8), the mass fractions of the unknowns are eliminated from the 
mass-balance equations in this summation. Darcy's law (2) may be substituted into the equations, and the equations may be written in terms of capillary pressure between the phases to yield:

$$
\begin{aligned}
n & {\left[\frac{\partial s_{\alpha}}{\partial P_{\mathrm{ow}}} \frac{\partial P_{\mathrm{ow}}}{\partial t}+\frac{\partial s_{\alpha}}{\partial P_{\mathrm{wg}}} \frac{\partial P_{\mathrm{wg}}}{\partial t}\right]+n s_{\alpha} \beta_{\alpha} \frac{\partial P^{\alpha}}{\partial t}+s_{\alpha} n \beta_{\mathrm{m}} \frac{\partial}{\partial t} P_{\mathrm{ave}} } \\
& -\nabla \cdot\left[\frac{\boldsymbol{k} k_{\mathrm{r} \alpha}}{\mu^{\alpha}} \cdot\left(\nabla P^{\alpha}-\rho^{\alpha} \boldsymbol{g}\right)\right]-\frac{1}{\rho^{\alpha}}\left[\frac{\boldsymbol{k} k_{\mathrm{r} \alpha}}{\mu^{\alpha}} \cdot\left(\nabla P^{\alpha}-\rho^{\alpha} \boldsymbol{g}\right)\right] \cdot \nabla \rho^{\alpha}= \\
& \frac{E^{\alpha}}{\rho^{\alpha}}-\frac{n s_{\alpha}}{\rho^{\alpha}} \frac{\partial \rho^{\alpha}}{\partial_{\omega}} \frac{\partial \omega}{\partial t}, \quad \alpha=\mathrm{o}, \text { w or } \mathrm{g}
\end{aligned}
$$

where $n=$ porosity of matrix (dimensionless); $P_{\text {ave }}=$ average fluid pressure in the pore space $\left[\mathrm{M} \mathrm{L}^{-1} \mathrm{~T}^{-2}\right] ; \beta_{\alpha}=$ compressibility of the $\alpha$ phase $\left[\mathrm{L} \mathrm{T}^{2} \mathrm{M}^{-1}\right]$; and $\beta_{\mathrm{m}}=$ compressibility of the matrix $\left[\mathrm{L} \mathrm{T}^{2} \mathrm{M}^{-1}\right]$.

In this paper, a two-mobile-phase formulation is used as the basis for a numerical model. This formulation fully describes a two-phase flow system such as the infiltration of an organic liquid into the saturated zone. For systems with more than two fluids, the application of two fluid-phase balance equations implies that some of the phases are either immobile or offer no inertial resistance to phase motion. The latter assumption is often applied to the soil-gas phase, since the viscosity of the gas phase is much smaller than the viscosity of a liquid phase (Abriola and Pinder, 1985a, b; Kaluarachchi and Parker, 1990). Thus, the ability to simulate the advective transport of volatile organic components in the gas phase is lost by this approach. Alternatively, one of the liquids in a three-phase regime may be treated as immobile. Sleep and Sykes (1990), Ryan and Cohen (1991), and Reeves (1993) used a two-mobile-phase formulation to study the influence of gas-phase mobility on the simulation of organic-phase infiltration and organic species transport. In these studies, the saturation of the water phase was assumed to be at or below residual levels. Numerical solutions for multicomponent transport in systems with three mobile phases have been discussed by Dorgarten and Tsang (1990), Mayer and Miller (1990), and Katyal et al. (1991).

The set-iterative compositional formulation employs a kinetic expression to describe the mass exchange between phases. The species-balance equations are derived directly from the general mass-balance equation (1) with substitution of the linear mass-exchange description (7) for the general mass-exchange terms, and of Fick's law (3) for the non-advective terms:

$$
\begin{aligned}
& \rho^{\alpha} n s_{\alpha} \frac{\partial \omega_{i}^{\alpha}}{\partial t}+\rho^{\alpha} n s_{\alpha} \boldsymbol{v}^{\alpha} \cdot \nabla \omega_{i}^{\alpha}-\nabla \cdot\left(n s_{\alpha} \rho^{\alpha} \boldsymbol{D}_{i}^{\alpha} \nabla \omega_{i}^{\alpha}\right)+k_{i}^{\alpha \beta} \omega_{i}^{\alpha}+\omega_{i}^{\alpha} E^{\alpha}=k_{i}^{\alpha \beta} \omega_{i}^{\alpha^{*}}, \\
& \quad \alpha=\mathrm{o}, \mathrm{w}, \mathrm{g}
\end{aligned}
$$


These equations are subject to proper initial and boundary conditions, and one of the phase velocities must be identically zero. Similar formulations have been proposed by Mayer and Miller (1990), and Guarnaccia and Pinder (1991). The mass fraction constraint (8) holds for each phase in this formulation. There are two unknown pressures and $3 N_{\mathrm{s}}$ unknown mass fractions. Thus, the model equations consist of 2 phase-balance equations (12), $3\left(N_{\mathrm{s}}-1\right)$ transport equations (13) and 3 mass fraction constraints (8).

In contrast to the kinetic formulation described above, an alternative computationally decoupled formulation maybe derived by assuming equilibrium partitioning between the phases (Kaluarachchi and Parker, 1990; Reeves, 1993). In this formulation, the mass-balance equation (1) is summed over all phases to derive a species-balance equation for each unknown chemical species. According to constraint (11), the source terms due to mass exchange between phases sum to zero in the species-balance equations. The summed equations, however, contain divergence of velocity terms that are difficult to approximate using standard finite-difference or linear finite-element techniques. These terms are typically eliminated through substitution of the phase-balance equation (12) into the summed species-balance equations to yield (Reeves and Abriola, 1988; Kaluarachchi and Parker, 1990):

$$
\begin{gathered}
\sum_{\alpha}\left[\rho^{\alpha} n s_{\alpha} \frac{\partial \omega_{i}^{\alpha}}{\partial t}+\rho^{\alpha} n s_{\alpha} \mathbf{v}^{\alpha} \cdot \nabla \omega_{i}^{\alpha}-\nabla \cdot\left(n s_{\alpha} \rho^{\alpha} D_{i}^{\alpha} \nabla \omega_{i}^{\alpha}\right)\right]= \\
\sum_{\alpha}-\omega_{i}^{\alpha} E^{\alpha}, \quad i=1, \ldots, N_{\mathrm{s}}-1
\end{gathered}
$$

Only $\left(N_{\mathrm{S}}-1\right)$ equations of this form need to be solved, as the remaining mass fraction can be obtained from the mass fraction constraint (8). Partitioning relationships (6) close the equation set.

Note that the procedure to eliminate the divergence of velocity terms reintroduces source terms in the summed equations. An iterative procedure between the species-balance equations (14) and the general mass-balance equations (1) is required to determine the magnitude of these non-linear terms. This type of iterative solution procedure leads to numerical difficulties during the simulation of flow with partitioning between the phases. There are two sources of error. The first is discussed by Kaluarachchi and Parker (1990). The iterative scheme to calculate the mass-exchange terms requires derivatives of phase velocity and estimates of the dispersive fluxes across the boundary. If the flow scenario produces sharp infiltration fronts, the derivatives of velocity may be difficult to estimate. Errors in the iterative scheme may be larger than the true mass-exchange terms introducing error into the phase- and speciesbalance equations. To avoid errors resulting from the iterative scheme, Kaluarachchi and Parker (1990) neglected the mass-exchange terms during 
times of high organic flow and, during the remainder of the simulation, corrected the computed mass-exchange terms by an independently computed mass-balance error. Their test used to determine whether a flow rate is "high" is that the velocity at a node exceeds the saturated organic conductivity at the node times 0.0001 .

A second source of error is discussed by Reeves (1993). As the equilibrium mass partitioning coefficients for the water and gas phases are reduced, the summed species-balance equation approaches the species-balance equation for the organic phase only. If dispersion within the organic phase has not been considered or is very small, a hyperbolic or nearly hyperbolic equation results. Approximation of a hyperbolic equation by a Galerkin finite-element method or centered finite-difference technique leads to oscillations or overshoot in the region of the front (Lapidus and Pinder, 1982). The oscillations will be incorporated into the estimation of the mass-exchange terms in the iterative solution of the species-balance equations. These incorrect massexchange terms are then passed to the phase-balance equations. Techniques applied to damp oscillations introduce artificial dispersion to the solution and the impact of this added error on the computed mass-exchange terms cannot be quantified or controlled. Use of the set-iterative compositional formulation eliminates the need for iterative calculation of the mass-exchange terms and the associated errors highlighted above. Mass-exchange terms are computed explicitly from the mass fractions via Eqs. 7 and 10.

The set-iterative model presented herein is based on the streamline-upwind Petrov-Galerkin finite-element method (Hughes and Brooks, 1982) to allow for upstream weighting of the phase- and species-balance equations. The form of this weighting function is:

$$
M_{i}=N_{i}+p \frac{\mathrm{d} N_{i}}{\mathrm{~d} x}
$$

where $M_{i}$ is the Petrov-Galerkin weighting function; $N_{i}$ is the standard linear weighting function; and $p$ is the Petrov-Galerkin weighting coefficient. The values of $p$ used in the simulations presented in this paper are given in Appendices A-C. Note that a standard Galerkin approximation is recovered when the Petrov-Galerkin weighting coefficient is set to zero. A consistent finite-element approach for the coefficients of the time derivatives is used, but the model has the option to lump the time derivative coefficient matrix in order to increase the stability of a solution. Following standard finite-element procedures, the integrations are performed on the local level, and Green's theorem is applied to the second-order term to reduce the order of differentiation and allow for the use of linear elements. Integration of the weak form of the equations yields a set of non-linear ordinary differential equations that is solved by approximating the time derivative with the 
variable-weight finite-difference method and iterating by successive substitution.

In the same manner, the weak form of the species-balance equations is integrated to give the semi-discrete matrix equations, and a variable time weight finite-difference technique is used to approximate the temporal derivatives in the semi-discrete equations. These equations may be nonlinear due to the mass-exchange terms and to the compositional dependence of terms in the coefficient matrices. In general, due to the mass-exchange terms, the species-balance equations are coupled. However, the coupled terms are lagged one iteration in the iterative procedure, and the equations are solved sequentially starting with the most volatile compound and proceeding to the least volatile. Sequential solution results in a set of smaller matrix equations requiring less computational burden for solution.

Convergence for a primary unknown is tested by applying the constraint:

$\delta \leqslant \frac{\operatorname{maximum}|\Delta \boldsymbol{u}|}{\left(\sum \boldsymbol{u}^{2}\right)^{1 / 2}}$

Here the maximum change in the primary variable over an iteration is normalized by a measure of the magnitude of the primary variable. The values of $\delta$ used for the species- and phase-balance equation sets for each simulation is given in Appendices A-C. The set-iterative compositional algorithm is summarized in Fig. 1.

As discussed above, when a kinetic expression is used to describe mass exchange, the mass-exchange terms are calculated directly and the ad hoc iterative solution described previously is not required. An additional benefit of employing a kinetic expression instead of the local equilibrium assumption is that the resulting numerical model may be used to explore non-equilibrium partitioning between phases. As expressions to describe the kinetic nature of mass exchange between phases in the subsurface are developed and additional data are measured, these may be directly included in the set-iterative compositional model. The penalty imposed by using a kinetic expression is an increase in computational burden. Mass-balance equations describing the transport of each species in each phase must be solved in place of one summed species mass-balance equation for each species.

\section{APPLICATION OF MODEL AND DISCUSSION}

\section{Mathematical verification}

Simulations on one-dimensional and two-dimensional domains are presented in this section to illustrate the capabilities of the set-iterative com- 


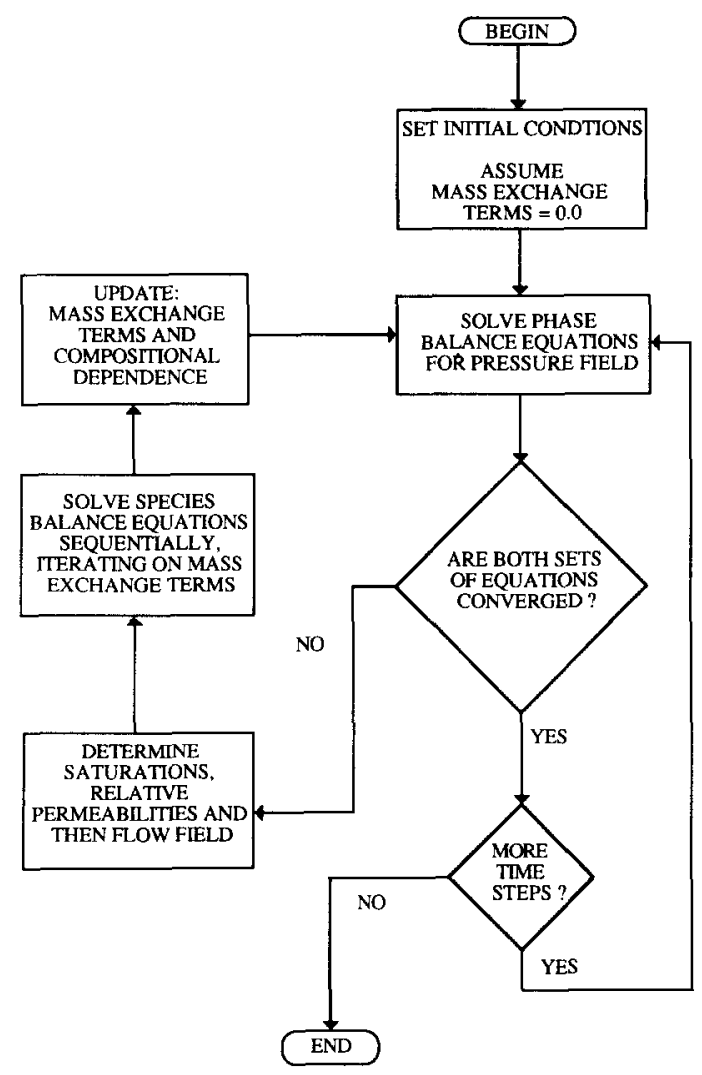

Fig. 1. Flow chart summarizing set-iterative compositional algorithm.

positional formulation and to compare performance of a numerical model based on this formulation to one based on a simultaneous formulation. The first problem studied is the one-dimensional infiltration of trichloroethylene into a fully saturated domain. This example is based on a problem simulated by Abriola (1984). Equilibrium partitioning of trichloroethylene into the water phase is assumed. Appendix A summarizes the physical and chemical data and the constitutive relationships required to describe this problem. Note that to simulate the infiltration of a single-component organic phase, the simultaneous formulation requires the introduction of an inert component. This inert component is assigned the same physical properties as trichloroethylene except that it is not allowed to partition to the aqueous phase. Both the simultaneous and set-iterative models used for this comparison require the establishment of organic-phase saturation throughout the domain. A small organic-phase saturation, $s_{\mathrm{o}}=0.0001$, composed entirely of the inert component is set as the initial condition. A mixture of trichloroethylene and the inert component is introduced at the boundary of the domain, and the 
partitioning coefficient for trichloroethylene is adjusted to give the proper concentration in the aqueous phase at the boundary.

Two options are used for the set-iterative model. Both options employ fully implicit weighting of the coefficient matrices for the approximation of the temporal derivatives:

(1) Option one involves the simulation of a two-component mixture mimicking the problem solved by the simultaneous formulation. Since there is no dispersion in the organic phase, upstream weighting is used in the setiterative model to circumvent numerical problems. The upstream weighting parameter for the species-balance equations is set to $\Delta \times / \sqrt{15}$. With the low solubility of trichloroethylene, the numerical model experiences some oscillatory behavior. Upstream weighting by the streamline-upwind technique helps to eliminate the unacceptable oscillations without adding a great deal of numerical dispersion to the result.

(2) The second option involves solving the trichloroethylene infiltration problem as a single-component problem. Employing this option reduces the number of species transport equations solved numerically since the organic phase is entirely trichloroethylene. The set-iterative formulation allows for simulation of a single-component organic if mass exchange from the organic phase to the other phases is set to zero when the phase saturation is below a critical value. The critical saturation value used in the simulations presented is 0.005 . For this option, the upstream weighting parameter was set to zero giving a standard Galerkin approximation for the species-balance equations.

Results from a finite-difference model based on a simultaneous formulation (Abriola and Pinder, 1985b) and the two options from the set-iterative compositional model are presented in Figs. 2 and 3.

Examination of the simulation results reveals that both models predict a sharp organic saturation front and a very sharp front for the aqueous-phase mass fraction of trichloroethylene. The mass fraction front does not extend a great deal into the domain for this situation since there is no externally imposed water-phase movement and the dispersive transport in the water phase is low. In contrast, Ryan and Cohen (1991) show contaminant transport fronts that extend well beyond the saturation front into an unsaturated domain. These fronts appear to be dominated by a large amount of dispersive transport in the soil-gas phase. The importance of dispersive transport away from the infiltrating organic liquid cannot be generalized, as it depends on the fluids present, the properties of the porous medium and the time scale of the simulations. In addition to the close agreement between simulation results for the two formulations, independent mass-balance calculations were performed to ensure that the models were solving the governing equations correctly. Both of the models produced 


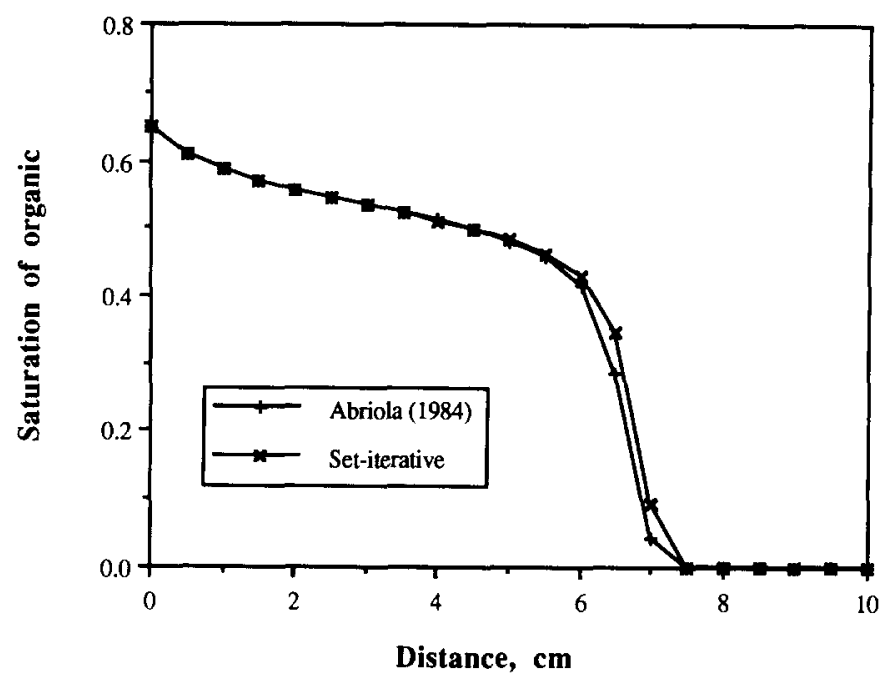

Fig. 2. Organic-phase saturation profiles for the infiltration of trichloroethylene into a saturated domain. Simulation results from the set-iterative compositional model and a model based on the simultaneous formulation (Abriola, 1984).

overall mass-balance results with errors less than $\pm 5 \%$ for the simulations shown. Numerical evaluation of mass-balance errors for multiphase finiteelement and finite-difference models is discussed by Abriola and Rathfelder (1993).

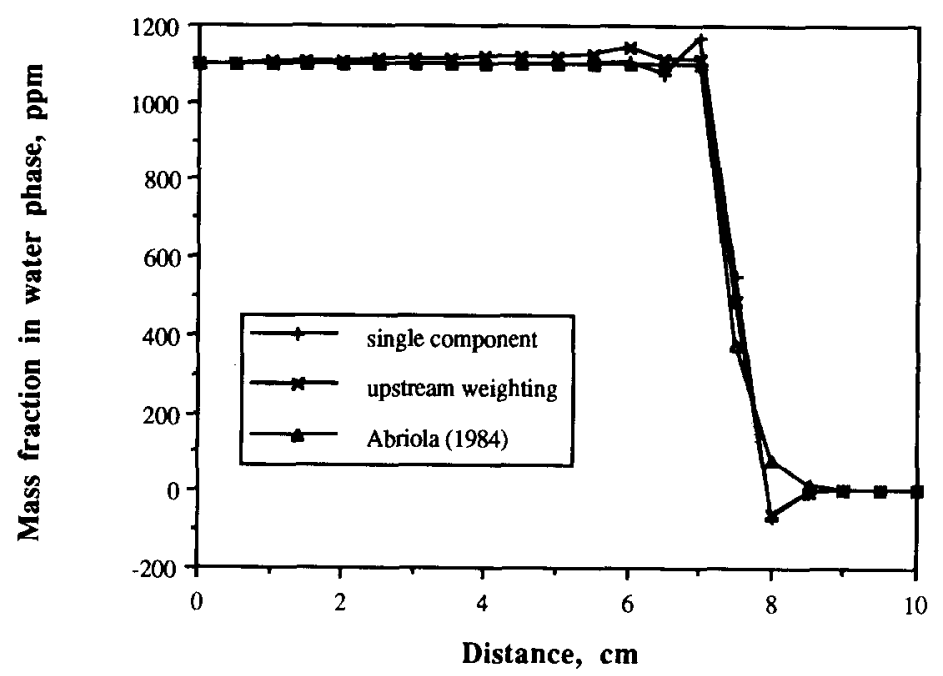

Fig. 3. Mass fraction of trichloroethylene in the water-phase profiles for the infiltration of trichloroethylene into a saturated domain. Simulation results from the set-iterative compositional model using a singlecomponent formulation and a two-component, upstream-weight technique and a model based on the simultaneous formulation (Abriola, 1984). 
TABLE 1

Operations per iteration for numerical models

\begin{tabular}{lll}
\hline Operation & Set-iterative FEM & Simultaneous FD \\
\hline$+/-$ & $\begin{array}{l}\text { phase balance: } 54\left(2 n_{\mathrm{n}}-1\right)+8\left(2 n_{\mathrm{n}}-3\right)+4 \\
\text { species balance: } n_{\text {trans }}\left(6 n_{\mathrm{n}}-5\right)\end{array}$ & $112\left(3 n_{\mathrm{n}}-1\right)+12\left(3 n_{\mathrm{n}}-5\right)+4$ \\
$* / \div \quad$ & $\begin{array}{l}\text { phase balance: } 14\left(2 n_{\mathrm{n}}-1\right)+6\left(2 n_{\mathrm{n}}-3\right)+2 \\
\text { species balance: } n_{\text {trans }}\left(6 n_{\mathrm{n}}-5\right)\end{array}$ & $32\left(3 n_{\mathrm{n}}-1\right)+10\left(3 n_{\mathrm{n}}-5\right)+2$ \\
& $\begin{array}{l}\text { Trichloroethylene test with inert component }\left(n_{\mathrm{n}}=21, n_{\text {trans }}=3\right): \\
+/-\end{array}$ & $\begin{array}{l}\text { phase balance: } 2,530 \\
\text { species balance: } 363 \\
\text { total: } 2,893\end{array}$ \\
& $\begin{array}{l}\text { phase balance: } 810 \\
\text { species balance: } 363 \\
\text { total: } 1,173\end{array}$ & 2,818 \\
\hline
\end{tabular}

FEM $=$ finite-element method; $F D=$ finite difference; $n_{n}=$ number of nodes; $n_{\text {trans }}=$ number of transport equations solved.

\section{Efficiency}

One of the proposed advantages of the set-iterative compositional formulation is that this formulation yields a more efficient numerical model. The computational effort used to solve the trichloroethylene comparison is presented to illustrate that the set-iterative compositional model does meet this expectation. The simultaneous formulation is solved using a finite-difference method with full Newton-Raphson iteration by Abriola (1984). Direct comparison of run times may not indicate true model performance since the Jacobian matrices required by the Newton-Raphson method are computationally burdensome and the set-iterative compositional formulation was solved without this added expense. Additionally for equivalent sets of equations, the Newton-Raphson iteration technique may allow for larger time steps, resulting in less total effort. Operations required to solve the matrix equations generated are therefore examined to assess model efficiency. Table 1 presents the operations required to solve the matrix equations arising from each formulation per iteration and the iterations required per time step for the trichloroethylene test problem. The number of iterations required to produce the profiles shown in Figs. 2 and 3, the number of nodes used in the test domain and the operation count in Table 1 may be used to compute the total number of operations required for the simulations and thereby indicate the overall efficiency of solution for this 
TABLE 2

Total operations required for solution of trichloroethylene simulations

\begin{tabular}{|c|c|c|c|}
\hline Operation & Set-iterative FEM & Simultaneous FD & $\begin{array}{l}\text { Savings } \\
(\%)\end{array}$ \\
\hline
\end{tabular}

Trichloroethylene test with inert component (water-, organic- and gas-phase species-balance equations solved; $n_{\mathrm{n}}=21, n_{\text {trans }}=3$ ):

\begin{tabular}{|c|c|c|c|}
\hline$+1-$ & $\begin{array}{l}\text { flow: } 5.2 \cdot 10^{6} \\
\text { species: } 0.6 \cdot 10^{6} \\
\text { total: } 5.8 \cdot 10^{6}\end{array}$ & $45 \cdot 10^{6}$ & 87 \\
\hline$* / \div$ & $\begin{array}{l}\text { flow: } 1.6 \cdot 10^{6} \\
\text { species: } 0.6 \cdot 10^{6} \\
\text { total: } 2.2 \cdot 10^{6}\end{array}$ & $15 \cdot 10^{6}$ & 85 \\
\hline
\end{tabular}

Single component test (organic-phase species-balance equation not considered; $n_{\mathrm{n}}=21$, $n_{\text {trans }}=2$ ):

\begin{tabular}{|c|c|c|c|}
\hline$+1-$ & $\begin{array}{l}\text { flow: } 5.4 \cdot 10^{6} \\
\text { species: } 0.2 \cdot 10^{6} \\
\text { total: } 5.6 \cdot 10^{6}\end{array}$ & $45 \cdot 10^{6}$ & 87 \\
\hline$* / \div$ & $\begin{array}{l}\text { flow: } 1.7 \cdot 10^{6} \\
\text { species: } 0.2 \cdot 10^{6} \\
\text { total: } 1.9 \cdot 10^{6}\end{array}$ & $15 \cdot 10^{6}$ & 87 \\
\hline
\end{tabular}

$F E M=$ finite-element method; $F D=$ finite difference.

problem. Table 2 presents the total number of operations and the savings gained by using the set-iterative formulation. The savings reported are the difference between the number of iterations required by the simultaneous model and the set-iterative model relative to the total required by the simultaneous model. Note that the single-component option saves some computational effort compared to the two-component option since the organicphase species-balance equation is not solved. This option should be used when modeling single-component organic phases since numerical difficulties associated with a hyperbolic transport equation discussed previously are also avoided.

Table 2 supports the claim that the set-iterative formulation leads to a relatively efficient numerical model. Examination of the operation counts tabulated for the comparison trials and the operations per iteration in Table 1 reveals that the savings realized by the set-iterative compositional model are greater than anticipated from the operations per iteration estimate. The anticipated savings for the two-component option are $63 \%$ for additions and subtractions, and $54 \%$ for multiplications and divisions. The 
reported savings for the simulations presented are $87 \%$ and $85 \%$, respectively. The difference is due to the total number of iterations required by the models to produce the results shown in Figs. 2 and 3. The simultaneous finitedifference model required 5945 iterations to achieve the solution at $72.25 \mathrm{~s}$ of simulation time. The set-iterative compositional model required 2041 iterations for the phase-balance equations and 1707 equations for the species-balance equations. Although the simultaneous formulation uses a Newton-Raphson iteration scheme, the successive-substitution iteration scheme for the set-iterative formulation allowed for larger time steps. The required time step size for the simultaneous formulation may be smaller because the primary variables, capillary pressure and mass fraction, are of drastically different orders of magnitude. The set-iterative approach separates solution of these variables and appears to yield sets of matrix equations that are easier to solve.

\section{Multicomponent chemistry}

In this section, the use of the set-iterative formulation to model the infiltration of a multicomponent organic mixture is demonstrated. Recall that the set-iterative formulation provides a more convenient framework for multicomponent simulations than the simultaneous formulation. The scenario simulated is a hypothetical column experiment where a solvent mixture is introduced to a vertical sand column with an imposed gradient on the water phase. In this simulation, two physical processes are examined: advective transport in the water phase and infiltration of an organic phase. The organic mixture is composed of three common solvents: methyl-ethyl ketone (2-butanone), trichloroethylene and tetrachloroethylene. Data for the simulations presented in this section are summarized in Appendix B. These simulations were performed using a fully implicit weighting for approximation of the time derivatives, consistent mass matrices and no upstream weighting.

Three different approaches to modeling transport of this organic mixture are considered. Mixtures 1 and 2 treat the organic phase as a two-component mixture with one inert component and one volatile component, as required by a simultaneous formulation with two components. Mixture $l$ is comprised of trichloroethylene and an inert mixture of tetrachloroethylene and methylethyl ketone. Mixture 2 consists of tetrachloroethylene as its inert component and a blend of trichloroethylene and methyl-ethyl ketone as its volatile component. The final mixture considers all of the components independently. The physical parameters for each mixture are calculated on the basis of a mixture containing $40 \%$ trichloroethylene, $45 \%$ tetrachloroethylene and $15 \%$ methylethyl ketone by weight. The purpose of modeling these three mixtures is to 


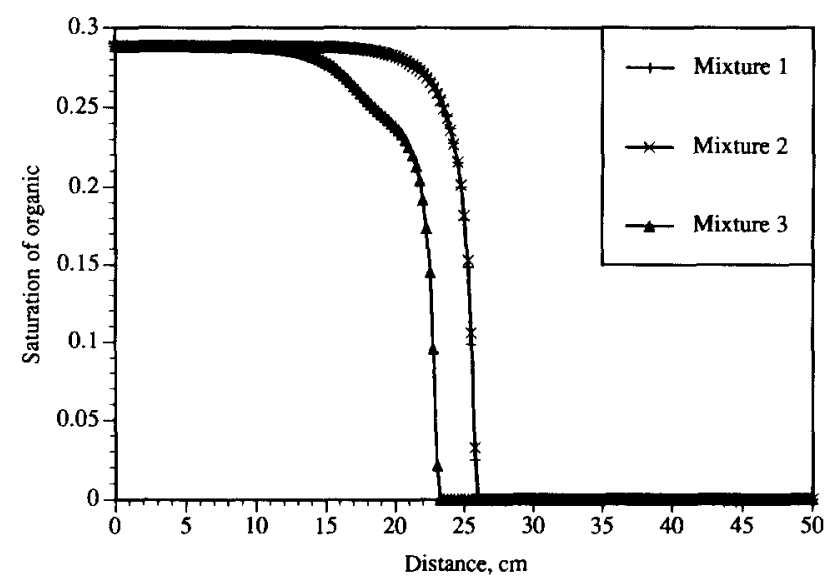

Fig. 4. Organic saturation profiles for the three mixtures after $6 \mathrm{~h}$ of simulation time.

examine the effect of the volatile methyl-ethyl ketone on the simulation of trichloroethylene transport in the moving aqueous phase. Mixtures 1 and 2 may be solved with existing simultaneous models, but these models cannot capture the chemical nature of the three-component solvent mixture.

Figs. 4 and 5 show the organic saturation fronts and the trichloroethylene mass fraction profiles at $6 \mathrm{~h}$ of simulation time. Clearly, the simulated behavior of trichloroethylene is altered when the full three-component mixture is modeled. In the simulations presented, the physical properties of the water phase are not altered by the dissolution of methyl-ethyl ketone. Thus, any differences in the profiles are solely due to partitioning effects. If the large amount of methyl-ethyl ketone changes the physical properties of the water phase, the differences between the modeling approaches would increase. Fig. 4

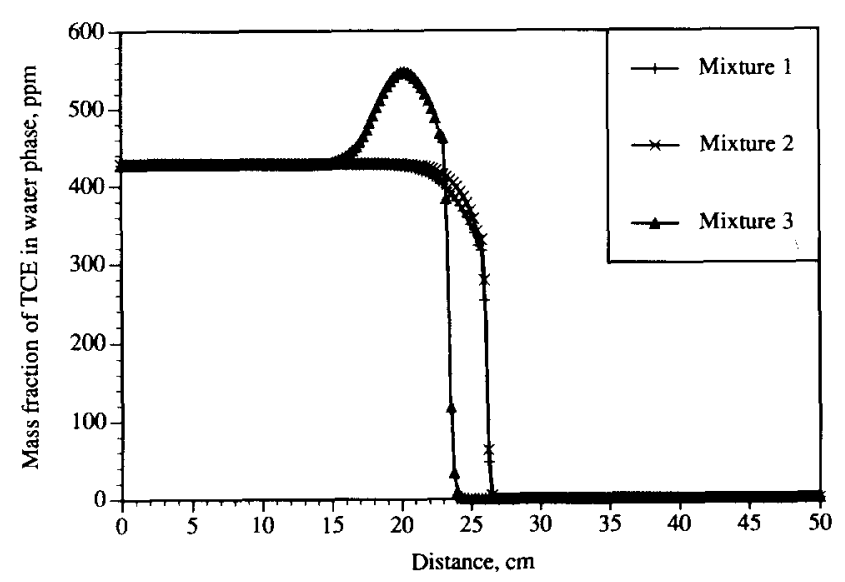

Fig. 5. Mass fraction profiles for trichloroethylene in the water phase for the three mixtures at 6 -h simulation time. 
reveals that the saturation front for the organic phase is affected as the mass of methyl-ethyl ketone partitions, reducing the amount of organic-phase mass in the domain. As the light-component partitions from the phase, the density and viscosity of the phase is altered, changing the flow behavior of the phase at the front. Fig. 5 reveals that, because methyl-ethyl ketone is much more soluble than trichloroethylene, the organic mixture becomes enriched in trichloroethylene at the saturation front. Equilibrium partitioning then predicts an elevation in trichloroethylene concentration in the water phase due to the enrichment in the organic phase. This type of behavior has also been observed in single-phase simulations where multicomponent organic mixtures are allowed to partition into a moving water or gas phase (Baehr, 1987). As simulation time and travel distance increases, the tendency for the organic phase to become enriched in less volatile components will also increase, leading to potentially high equilibrium concentrations in the water phase. The importance of this enrichment and subsequent elevation of trichloroethylene concentrations in the aqueous phase lies in the potential exposure to trichloroethylene at concentrations higher than predicted from the source mixture properties.

\section{Effect of mass-transfer rates}

All of the simulations presented in the previous sections have used a high value for the mass-exchange coefficient to mimic equilibrium partitioning. They also have been performed on one-dimensional domains. In this section, a two-dimensional finite-element approximation to the governing equations is used to show the effect of varying mass-transfer coefficients on the transport of trichloroethylene into a saturated domain. Linear triangular elements and a simple direct solver are used in the two-dimensional numerical model. Details of the two-dimensional approximations are presented by Reeves (1993). The domain characteristics and physical parameters used for the simulations presented in this section are summarized in Appendix C.

Powers et al. (1991) presented a theoretical investigation of the effect of mass-transfer limitations on the dissolution of entrapped trichloroethylene in a sand aquifer. In this example simulation, trichloroethylene is introduced into a saturated domain and water is allowed to flow through and around the advancing organic body. The lumped mass-transfer coefficient required by the numerical model is estimated from the discussion presented by Powers et al. (1991). This lumped mass-transfer coefficient may be described by:

$\bar{k}_{i}^{\mathrm{ow}}=\frac{k_{f}}{n s_{\mathrm{w}}}\left[n s_{\mathrm{o}}\left(\frac{A}{V}\right) f\right]$

where $k_{f}=$ mass-transfer coefficient $\left[\mathrm{L} \mathrm{T}^{-1}\right] ; A / V=$ area to volume ratio for 


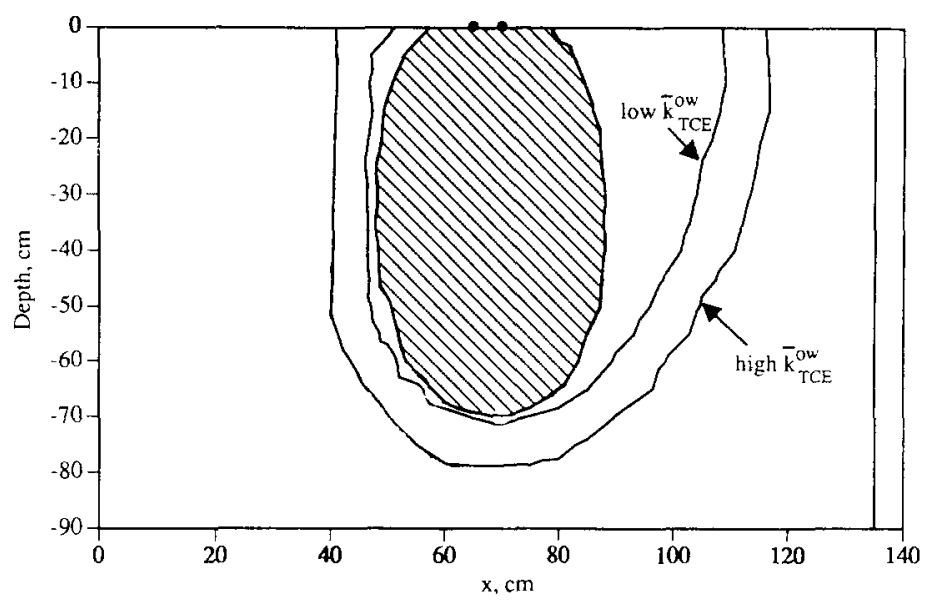

Fig. 6. Normalized mass fraction contours for trichloroethylene in the water phase at 12-h simulation time. Contour level is 0.2 . The shaded region corresponds to an organic-phase saturation contour of 0.1 .

the organic phase $\left[\mathrm{L}^{-1}\right]$; and $f=$ fraction of exposed area for the organic phase (dimensionless).

Following Powers et al. (1991), the exposed fraction is estimated as 0.1 and the area to volume ratio as $15 \mathrm{~cm}^{-1}$. The largest mass-transfer coefficient estimated by the authors is $9.9 \cdot 10^{-2} \mathrm{~cm} \mathrm{~s}^{-1}$. Setting the representative organic saturation as 0.1 allows calculation of the lumped mass-exchange coefficient through Eq. 17. The value using these parameters is $1.6 \cdot 10^{-2} \mathrm{~s}^{-1}$. To investigate the importance of this coefficient, the simulation was repeated with a lumped mass transfer two orders of magnitude smaller than this estimated value. This value is within the range of lumped mass-transfer coefficients estimated by Powers et al. (1991). Profiles for simulations with lumped mass-transfer coefficients equal to $1.6 \cdot 10^{-2}$ and $1.6 \cdot 10^{-4} \mathrm{~s}^{-1}$ are presented in Fig. 6 .

This figure, presented for a simulation time of $12 \mathrm{~h}$, illustrates the importance of the rate of mass transfer on aqueous-phase concentrations. The contours shown in Fig. 6 are for normalized aqueous-phase mass fractions of 0.2 for the two simulations. The normalization is computed relative to the solubility of trichloroethylene in water. For this simulation time and this pure organic phase, the mass transfer does not appreciably affect the mass of the saturation profile in the domain. The smaller value of the lumped masstransfer coefficient, however, produces aqueous-phase mass fraction profiles containing much less trichloroethylene and exhibiting less spread of the organic pollutant in the vicinity of the spill. These low aqueous-phase concentrations of trichloroethylene qualitatively support the observation that concentrations of organic solvents in the aqueous phase rarely approach their solubility values even if free product is present (Mackay et al., 1985). 
A great deal of research is presently focused on mass-transfer kinetics and rates for partitioning between organic pollutants and groundwater. This is an important issue, as it affects the interpretation of field measurements and the design of remediation alternatives. The set-iterative formulation presented in this paper may be used to simulate different kinetic expressions and can be an important tool in evaluating the importance of mass-transfer limitations in multiphase flow scenarios.

\section{SUMMARY}

Three distinct formulations of the partial differential equations describing multiphase flow with multicomponent transport in the subsurface have been presented. Each formulation offers inherent advantages and disadvantages for numerical approximation. The simultaneous formulation is the most straightforward and leads to a numerical approximation with less complications and iterative procedures. However, the matrix equations resulting from this formulation are large, and numerical models based on this formulation are not expected to be very efficient. The adaptive IMPES formulation address the inefficiencies of the simultaneous formulation by rearranging the equations to a multi-step solution procedure. Numerical models based on this formulation are expected to be more efficient due to smaller matrix equations and explicit evaluation of secondary unknowns. However, this approach may experience convergence problems due to the multi-step nature of the solution. The third formulation separates the problem along natural divisions by treating the fluid flow and species transport problems separately and iterating between the two equation sets. Again efficiency results from the solution of smaller equation sets. This computationally decoupled formulation also offers a framework for multicomponent extensions of a basic model without extensive reformulation and rewriting. Since an iterative procedure is required to determine the coupling between the flow and transport processes, numerical problems may result if the coupling becomes significant or if numerical errors mask the natural coupling between equation sets.

In this paper, the set-iterative compositional formulation describing multiphase flow with multicomponent transport has been introduced. Three major advantages of the set-iterative compositional formulation were illustrated through the use of simulation results. The first major advantage of this formulation is the ability to simulate non-equilibrium partitioning. A number of recent papers have suggested the importance of non-equilibrium partitioning to groundwater from non-aqueous-phase liquids. The presented model may be used to evaluate the impact of different kinetic expressions as they are proposed. The second major advantage of the model is that the formulation provides a flexible framework for multicomponent simulations. For mixtures 
of organic solvents, an understanding of these multicomponent effects may be important to estimate aqueous-phase compositions and bulk organic-phase movement. Finally, the set-iterative compositional formulation leads to increased efficiency due to generation of smaller matrix problems. This increased efficiency allows for feasible simulation of larger, more complex problems. Work is continuing on this model to increase the efficiency of the two-dimensional code by use of more powerful matrix solution techniques. The model will be applied to study the importance of domain heterogeneities on multiphase flow simulations. Results from laboratory experiments investigating the kinetics of mass-transfer partitioning may also be incorporated into the code to more fully investigate the impact of mass transfer on simulation results.

\section{ACKNOWLEDGEMENTS}

This work was supported in part by: the National Science Foundation Grant ECE-8451469 with matching funds and equipment from General Motors Corporation and Sun Microsystems, Inc.; and the National Institute of Environmental Health Sciences, Grant ES04911-01. The paper has not been subject to NSF or NIEHS review, and, thus, the views and opinions herein do not necessarily reflect those of NSF or NIEHS. A Ph.D. Student Loan from General Electric administered by The University of Michigan is also gratefully acknowledged.

\section{APPENDIX A - ONE-DIMENSIONAL INFILTRATION OF TRICHLOROETHYLENE}

The boundary conditions, initial conditions and data used for the numerical simulation of trichloroethylene infiltration into a one-dimensional domain are provided in Table A-1.

\section{APPENDIX B - DATA FOR ONE-DIMENSIONAL SIMULATION OF MULTICOMPONENT MIXTURES}

Three multicomponent mixtures are considered for the hypothetical simulations. Each simulation is run on a one-dimensional, vertical domain. The initial condition, however, imposes a gradient of 0.005 on the water phase to induce water-phase movement down the column. The constitutive relationships are held constant for each simulation as are the other physical and numerical data required by the model. The water-phase and matrix properties are the same as used in the first simulation summarized in Table A-1 except where listed in Table B-1. The other data that are held constant for the three mixtures are listed in Table B-1.

The three mixtures contain trichloroethylene, methyl-ethyl ketone (2-butanone) and tetrachloroethylene. The mass fractions for the three organic components are $0.4,0.15$ and 0.45 , respectively. Mixture 1 is a two-component organic phase, where the volatile phase is pure 
TABLE A-1

Parameters for one-dimensional TCE simulations

\begin{tabular}{|c|c|c|c|}
\hline Parameter & Value & Parameter & Value \\
\hline Domain: & \multicolumn{3}{|c|}{ Trichloroethylene: } \\
\hline$L$ & $10 \mathrm{~cm}$ & $M_{\mathrm{o}}$ & $131.4 \mathrm{~g} \mathrm{~mol}^{-1}$ \\
\hline$\Delta x$ & $0.5 \mathrm{~cm}$ & $\mu_{\mathrm{o}}$ & $5.8 \cdot 10^{-3} \mathrm{P}$ \\
\hline$\Delta t$ & $0.001-0.25 \mathrm{~s}$ & $\beta_{0}$ & $0.0 \mathrm{~cm}^{2} \mathrm{dyn}^{-1}$ \\
\hline Simulation time & $72.25 \mathrm{~s}$ & $\rho^{\circ}$ & $1.4657 \mathrm{~g} \mathrm{~cm}^{-3}$ \\
\hline$T$ & $293.15 \mathrm{~K}$ & $P_{\text {ref }}^{\mathrm{o}}$ & $1.0133 \cdot 10^{6} \mathrm{dyn} \mathrm{cm}^{-2}$ \\
\hline$g$ & $980.665 \mathrm{~cm} \mathrm{~s}^{-2}$ & & \\
\hline$R$ & $\begin{array}{l}8.3157 \cdot 10^{7} \mathrm{dyn} \\
\mathrm{cm} \mathrm{mol}{ }^{-1} \mathrm{~K}^{-1}\end{array}$ & Air: & \\
\hline Water: & & $M_{\mathrm{g}}$ & $28.97 \mathrm{~g} \mathrm{~mol}^{-1}$ \\
\hline$M_{\mathrm{w}}$ & $18.02 \mathrm{~g} \mathrm{~mol}^{-1}$ & & \\
\hline$\mu_{\mathrm{w}}$ & $1.0019 \cdot 10^{-2} \mathrm{P}$ & Matrix: & \\
\hline$\beta_{w}$ & $4.531 \cdot 10^{-11} \mathrm{~cm}^{2} \mathrm{dyn}^{-1}$ & $\begin{array}{l}\mathrm{n} \\
\beta_{\mathrm{m}}\end{array}$ & $\begin{array}{l}0.36 \\
2.0 \cdot 10^{-10} \mathrm{~cm}^{2} \mathrm{dyn}^{-1}\end{array}$ \\
\hline$\rho^{\mathrm{w}}$ & $1.0 \mathrm{~g} \mathrm{~cm}^{-3}$ & $k$ & $5.8231 \cdot 10^{-7} \mathrm{~cm}^{2}$ \\
\hline$P_{\mathrm{ref}}^{\mathrm{w}}$ & $1.0133 \cdot 10^{6} \mathrm{dyn}^{-2}$ & & \\
\hline
\end{tabular}

Partitioning:

Dispersion and diffusion:

Solubility of TCE $1,100 \mathrm{ppm}$

$\begin{array}{lll}\left(1.5 \cdot 10^{-4} \mathrm{~mol} \mathrm{~mol}^{-1}\right) & D^{\mathrm{mw}} & 8.434 \cdot 10^{-6} \mathrm{~cm}^{2} \mathrm{~s}^{-1} \\ & D^{\mathrm{mo}} & 0.0 \mathrm{~cm}^{2} \mathrm{~s}^{-1} \\ & a^{\mathrm{w}} & 0.1 \mathrm{~cm} \\ a^{\mathrm{o}} & 0.0 \mathrm{~cm}\end{array}$

Initial and boundary conditions:

Constitutive relationships:
$P_{\text {ow }}(x, 0)$
0.0
$P_{\mathrm{wg}}(x, 0)$
hydrostatic
$\omega_{\text {tce }}^{\mathrm{o}}(x, 0)$
$P_{\text {ow }}(0, t)$
$P_{\mathrm{wg}}(0, t)$
$\omega_{\mathrm{tce}}^{\mathrm{o}}(0, t)$
0.0
$\partial P_{\mathrm{ow}}(L, t) / \partial x$
$14,610 \mathrm{dyn}^{-2}$
$0.0 \mathrm{dyn}^{-2}$
1.0
0.0 dyn $\mathrm{cm}^{-3}$
$P_{\mathrm{wg}}(L, t)$
0.0 dyn $\mathrm{cm}^{-2}$
0.0
$s_{\mathrm{w}}=s_{\mathrm{wir}}+\frac{s_{\mathrm{swu}}-s_{\mathrm{wir}}}{\left[\left(1.0+\left(\alpha_{\mathrm{w}} H_{\mathrm{wg}}\right)^{\mathrm{sw}}\right)^{\mathrm{msw}}\right]}$
$s_{\mathrm{g}}$
$s_{\mathrm{o}}$
$s_{\text {wir }}$
0.0001
$\omega_{\mathrm{tce}}^{\mathrm{o}}(L, t)$
$s_{\text {swo }}$
$1.0-s_{\mathrm{w}}-s_{\mathrm{g}}$
0.306
$s_{\mathrm{sW}}$
0.9998
$n_{\mathrm{sW}}$
0.9998
$m_{\text {sw }}$
8.605
$k_{\mathrm{rw}}=\frac{\left[\left(1-a_{\mathrm{hw}} \mathrm{b}_{\mathrm{nW}-1}\right)\left(1+a_{\mathrm{hw}}\right)^{-b_{\mathrm{mw}}} / 2\right.}{\left(1+a_{\mathrm{hw}}^{b_{\mathrm{WW}}}\right)^{b_{\mathrm{mw}} / 2}}$
$\alpha_{w}$
$1-1 /$ nsw
$b_{\text {nw }}$
0.11
$k_{\mathrm{ro}}=0.4 \sqrt{\bar{s}}\left[1-\left(1-\bar{s}^{1 / \mathrm{b}_{\mathrm{mo}}}\right)^{\mathrm{b}_{\mathrm{mo}}}\right]^{2}$
$\bar{s} \quad 1-\left[\left(s_{\mathrm{w}}-s_{\mathrm{wir}}\right) /\left(s_{\mathrm{sw}}-s_{\mathrm{wir}}\right)\right]$
$b_{\mathrm{mo}}$
0.7059
6.6
$0.108\left(P_{\text {ow }} / \rho^{\mathrm{w}} g\right)$

$\mathrm{TCE}=$ trichloroethylene. 
TABLE B-1

Parameters for one-dimensional multicomponent simulations

\begin{tabular}{|c|c|c|c|}
\hline Parameter & Value & Parameter & Value \\
\hline \multicolumn{2}{|l|}{ Domain: } & \multicolumn{2}{|l|}{ Mixture $I$ : } \\
\hline \multirow{5}{*}{$\begin{array}{l}L \\
\Delta x \\
\Delta t \\
\text { Simulation time }\end{array}$} & $50 \mathrm{~cm}$ & \multirow{4}{*}{$\begin{array}{l}\omega_{\mathrm{i}}^{\mathrm{o}}(x, 0) \\
\omega_{\mathrm{tce}}^{\mathrm{o}}(0, t)\end{array}$} & 0.0 \\
\hline & $\begin{array}{l}50 \mathrm{~cm} \\
0.25 \mathrm{~cm}\end{array}$ & & \multirow[t]{3}{*}{0.4} \\
\hline & \multirow{2}{*}{$\begin{array}{l}0.1-5.0 \mathrm{~s} \\
21,600 \mathrm{~s}\end{array}$} & & \\
\hline & & & \\
\hline & \multicolumn{3}{|c|}{ Mixture 2: } \\
\hline \multirow{2}{*}{\multicolumn{2}{|c|}{ Initial and boundary conditions: }} & $\omega_{i}^{o}(x, 0)$ & 0.0 \\
\hline & & $\omega_{\text {tce } / \text { mek }}^{o}(0, t)$ & 0.55 \\
\hline \multirow{9}{*}{$\begin{array}{l}P_{\mathrm{ow}}(x, 0) \\
P_{\mathrm{wg}}(x, 0) \\
P_{\mathrm{ow}}(0, t) \\
P_{\mathrm{wg}}(0, t) \\
\partial P_{\mathrm{ow}}(L, t) / \partial x \\
P_{\mathrm{wg}}(L, t)\end{array}$} & 0.0 & & \\
\hline & head gradient 0.005 & \multirow{3}{*}{\multicolumn{2}{|c|}{ Mixture 3: }} \\
\hline & $14,610 \mathrm{dyn} \mathrm{cm}^{-2}$ & & \\
\hline & $490.3325 \mathrm{dyn} \mathrm{cm}^{-2}$ & & \\
\hline & $0.0 \mathrm{dyn}^{-3}$ & $\omega_{i}^{o}(x, 0)$ & 0.0 \\
\hline & $0.0 \mathrm{dyn} \mathrm{cm}^{-2}$ & $\omega_{\text {mek }}^{0}(0, t)$ & 0.15 \\
\hline & & $\omega_{\mathrm{tce}}^{\mathrm{o}}(0, t)$ & 0.4 \\
\hline & \multicolumn{3}{|c|}{ All mixtures: } \\
\hline & & $\omega_{i}^{0}(L, t)$ & 0.0 \\
\hline
\end{tabular}

trichloroethylene and the inert phase is a blend of tetrachloroethylene and methyl-ethyl ketone. Mixture 2 is a two-component organic phase, where the volatile phase is a blend of trichloroethylene and methyl-ethyl ketone and the inert phase is pure tetrachloroethylene. The solubility of the trichloroethylene/methyl-ethyl ketone blend is adjusted to give a water-phase mass fraction equal to that resulting from the partitioning of trichloroethylene from the other two mixtures. Finally, mixture 3 is a three-component organic phase. The molecular diffusion coefficients for each organic component are estimated using the Wilke-Chang correlation (Reid et al., 1977):

$D_{a b}=7.4 \cdot 10^{-8} \frac{\left(\phi M_{b}\right)^{1 / 2} T}{\mu_{b} V_{a}^{0.6}}$

where $D_{a b}=$ diffusion coefficient of component $a$ in solvent $b\left(\mathrm{~cm}^{2} \mathrm{~s}^{-1}\right) ; M_{b}=$ molecular weight of the solvent $b\left(\mathrm{~g} \mathrm{~mol}^{-1}\right) ; T=$ absolute temperature $(\mathrm{K}) ; \mu_{b}=$ viscosity of solvent $b$ (cP); $V_{a}=$ molal volume of solute $a$ at normal boiling temperature $\left(\mathrm{cm}^{3} \mathrm{~mol}^{-1}\right)$; and $\phi=$ association factor for solvent $b$.

The association factor was set to 2.6 for the aqueous phase and 1.0 for the organic phase. The molal volume for the organic solutes were estimated using the La Bas additive volumes (Reid et al., 1977). Mixture density and viscosity are estimated assuming ideal behavior:

$\rho^{\circ}=\frac{1}{\sum_{i=1}^{N_{\mathrm{s}}} \frac{\omega_{i}^{o}}{\rho_{t}}}$ 
and

$\mu^{\mathrm{o}}=\sum_{i=1}^{N_{\mathrm{s}}} \mu_{i}^{x_{i}}$

where $\omega_{i}^{o}=$ mass fraction of the $i$ th component in the mixture; and $x_{i}=$ mole fraction of the $i$ th component in the mixture.

The properties of each mixture are summarized in Table B-2.

\section{TABLE B-2}

Physical and chemical properties for mixture simulations

\begin{tabular}{|c|c|c|c|}
\hline Parameter & Value & Parameter & Value \\
\hline Mixture 1: & & Mixture 1: & \\
\hline Component $I$ & trichloroethylene & Component 2 & $\begin{array}{l}\text { MEK/ } \\
\text { tetrachloroethylene }\end{array}$ \\
\hline$M_{\mathrm{o}}$ & $131.4 \mathrm{~g} \mathrm{~mol}^{-1}$ & $M_{\mathrm{o}}$ & $125.2 \mathrm{~g} \mathrm{~mol}^{-1}$ \\
\hline$\mu_{\mathrm{o}}$ & $5.8 \cdot 10^{-3} \mathrm{P}$ & $\mu_{\mathrm{o}}$ & $6.59 \cdot 10^{-3} \mathrm{P}$ \\
\hline$\beta_{0}$ & $0.0 \mathrm{~cm}^{2} \mathrm{dyn}^{-1}$ & $\beta_{0}$ & $0.0 \mathrm{~cm}^{2} \mathrm{dyn}^{-1}$ \\
\hline$\rho^{0}$ & $1.4657 \mathrm{~g} \mathrm{~cm}^{-3}$ & $\rho^{\mathrm{O}}$ & $1.294 \mathrm{~g} \mathrm{~cm}^{-3}$ \\
\hline$P_{\text {ref }}^{o}$ & $1.0133 \cdot 10^{6} \mathrm{dyn}^{-2}$ & Solubility & $0 \mathrm{ppm}$ \\
\hline Solubility & $1,100 \mathrm{ppm}$ & & \\
\hline$D^{\mathrm{mw}}$ & $2.5 \cdot 10^{-6} \mathrm{~cm}^{2} \mathrm{~s}^{-1}$ & & \\
\hline$D^{\mathrm{mo}}$ & $6.1 \cdot 10^{-6} \mathrm{~cm}^{2} \mathrm{~s}^{-1}$ & & \\
\hline
\end{tabular}

Mixture 2:

Mixture 2:

$\begin{array}{cccc}\text { Component } 1 & \begin{array}{c}\text { trichloroethylene/ Component } 2 \\ \text { MEK }\end{array} & \text { tetrachloroethylene }\end{array}$

$\begin{array}{ll}M_{\mathrm{o}} & 107.3 \mathrm{~g} \mathrm{~mol}^{-1} \\ \mu_{\mathrm{o}} & 4.82 \cdot 10^{-3} \mathrm{P} \\ \beta_{\mathrm{o}} & 0.0 \mathrm{~cm}^{2} \mathrm{dyn}^{-1} \\ \rho^{\mathrm{o}} & 1.198 \mathrm{~g} \mathrm{~cm}^{-3} \\ \text { Solubility } & 655 \mathrm{ppm}^{\mathrm{o}} \\ D^{\mathrm{mw}} & 2.5 \cdot 10^{-6} \mathrm{~cm}^{2} \mathrm{~s}^{-1} \\ D^{\mathrm{mo}} & 5.1 \cdot 10^{-6} \mathrm{~cm}^{2} \mathrm{~s}^{-1}\end{array}$

Mixture 3:

Mixture 3:

\begin{tabular}{lll} 
Component 1 & MEK & Component 2 \\
$M_{\mathrm{o}}$ & $72.1 \mathrm{~g} \mathrm{~mol}^{-1}$ & \\
$\mu_{\mathrm{o}}$ & $4.2 \cdot 10^{-3} \mathrm{P}$ & \\
$\beta_{\mathrm{o}}$ & $0.0 \mathrm{~cm}^{2} \mathrm{dyn}^{-1}$ & Component 3 \\
$\rho^{\mathrm{o}}$ & $0.805 \mathrm{~g} \mathrm{~cm}^{-3}$ & \\
$P_{\mathrm{ref}}^{\mathrm{o}}$ & $1.0133 \cdot 10^{6} \mathrm{dyn} \mathrm{cm}^{-2}$ & \\
Solubility & $240,000 \mathrm{ppm}^{\mathrm{m}}$ & \\
$D^{\mathrm{mw}}$ & $2.5 \cdot 10^{-6} \mathrm{~cm}^{2} \mathrm{~s}^{-1}$ & \\
$D^{\mathrm{mo}}$ & $5.1 \cdot 10^{-6} \mathrm{~cm}^{2} \mathrm{~s}^{-1}$ & \\
\hline
\end{tabular}

$165.83 \mathrm{~g} \mathrm{~mol}^{-1}$

$9.3 \cdot 10^{-3} \mathbf{P}$

$0.0 \mathrm{~cm}^{2} \mathrm{dyn}^{-1}$

$1.623 \mathrm{~g} \mathrm{~cm}^{-3}$

0 ppm

$\mathrm{MEK}=$ methyl-ethyl ketone. 
SOURCE NODES - FIXED HEAD, FIXED MASS FRACTION
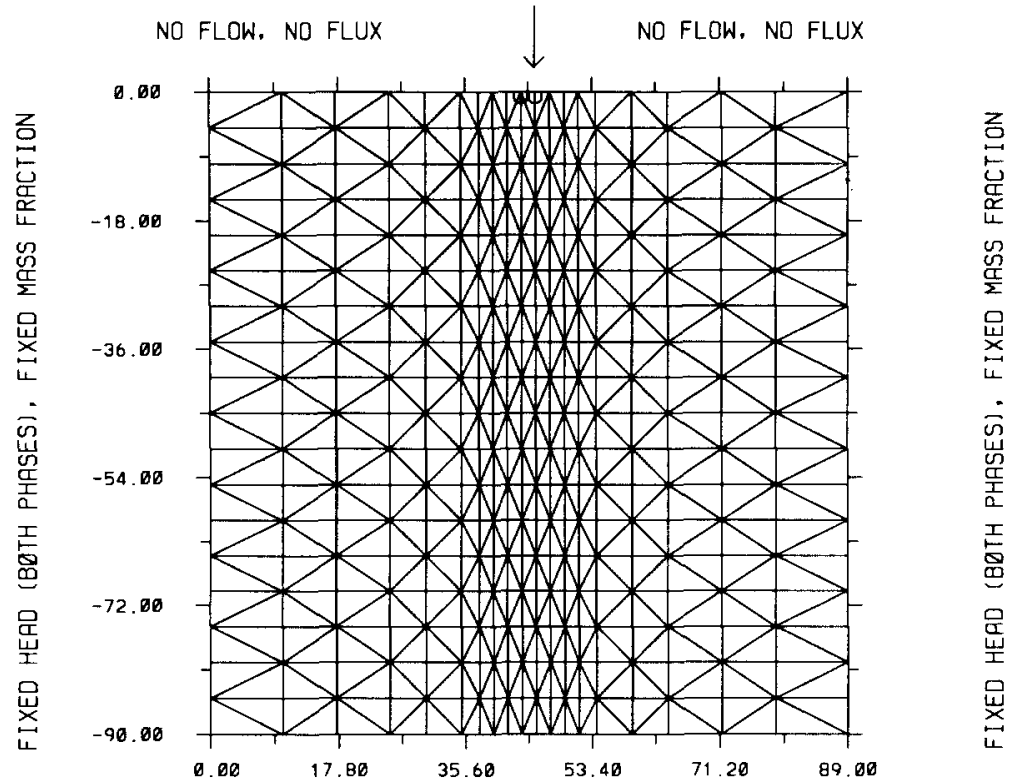

NO FLOW OF EITHER PHASE. NO FLUX OF COMPONENT

$\rightarrow$ WATER PHASE GRPDIENT $=0.005$

Fig. C-1. Two-dimensional domain, elements and boundary conditions.

\section{TABLE C-1}

Parameters for two-dimensional mass-exchange simulations

\begin{tabular}{ll}
\hline Parameter & Value \\
\hline $\begin{array}{l}\text { Domain } \\
\text { Number of nodes }\end{array}$ & 380 \\
Number of elements & $684 \Delta x$ \\
$\quad$ minimum & $5.0 \mathrm{~cm}$ \\
maximum & $10.0 \mathrm{~cm} \Delta z$ \\
minimum & $5.0 \mathrm{~cm}$ \\
maximum & $5.0 \mathrm{~cm}$ \\
$\Delta t$ & $10-600 \mathrm{~s}$, acceleration factor $=1.4$ \\
Simulation time & $42,300 \mathrm{~s}$ \\
Dispersivities & \\
$a_{1}^{\mathrm{w}}$ & $0.5 \mathrm{~cm}$ \\
$a_{\mathrm{t}}^{\mathrm{w}}$ & $0.05 \mathrm{~cm}$ \\
$a_{1}^{\mathrm{o}}$ & $0.5 \mathrm{~cm}$ \\
$a_{\mathrm{t}}^{\mathrm{o}}$ & $0.05 \mathrm{~cm}$ \\
\hline
\end{tabular}




\section{APPENDIX C - DATA FOR TWO-DIMENSIONAL SIMULATIONS TESTING MASS- EXCHANGE RATES}

The hypothetical domain with the applied boundary conditions and numerical grid is presented in Fig. C-1. The imposed gradient of the piezometric head of the water phase is 0.005 . The constitutive relationships are held constant for each simulation as are the other physical and numerical data required by the model. The water phase, trichloroethylene phase and matrix properties are the same as used in the simulations summarized in Tables B-1 and B-2 except where noted in Table $\mathrm{C}-1$. The constitutive relationships are also the same as used for the previous simulations summarized in Tables A-1 and B-1. The other data that are required for the simulations are listed in Table C-1.

\section{REFERENCES}

Abriola, L.M., 1984. Multiphase Migration of Organic Compounds in a Porous Media - A Mathematical Model. Springer, Berlin, $232 \mathrm{pp}$.

Abriola, L.M. and Pinder, G.F., 1985a. A multiphase approach to the modeling of porous media contamination by organic compounds, 1. Equation development. Water Resour. Res., 21(1): 11-18.

Abriola, L.M. and Pinder, G.F., 1985b. A multiphase approach to the modeling of porous media contamination by organic compounds, 2. Numerical simulation. Water Resour. Res., 21(1): $19-26$.

Abriola, L.M. and Rathfelder, K.M., 1993. Mass balance errors in modeling two-phase immiscible flows: Causes and remedies. Adv. Water Resour., 16: 223-239.

Baehr, A.L., 1987. Selective transport of hydrocarbons in the unsaturated zone due to aqueous and vapor phase partitioning. Water Resour. Res., 23(10): 1926-1938.

Bear, J., 1972. Dynamics of Fluids in Porous Media. American Elsevier, New York, NY, 764 pp. (re-issued by Dover, New York, NY, 1988).

Brusseau, M.L., 1992. Rate-limited mass transfer and transport of organic solutes in porous media that contain immobile immiscible organic liquid. Water Resour. Res., 28(1): 33-46.

Dorgarten, H.W. and Tsang, C.F., 1990. Three-phase simulation of organic contaminations in aquifer systems. IAH (Int. Assoc. Hydrogeol.) Conf. on Subsurface Contamination by Immiscible Fluids, Calgary, Alta., April 18-20, 1990, pp 149-158.

Falta, Jr., R.W. and Javendel, I., 1987. A numerical method for multiphase multicomponent contaminant transport in groundwater systems. Eos (Trans. Am. Geophys. Union), 68(44): 1284 (abstract).

Forsyth, Jr., P.A., 1988. Simulation of nonaqueous phase groundwater contamination. Adv. Water Resour., 11: 74-83.

Forsyth, Jr., P.A. and Sammon, P.H., 1986. Practical considerations for adaptive implicit methods in reservoir simulation. J. Comput. Phys., 62(2): 265-281.

Guarnaccia, J.F. and Pinder, G.F., 1991. Simulation of two phase flow and transport with interphase mass exchange. Eos (Trans. Am. Geophys. Union), 72(17): 120 (abstract).

Hassanizadeh, M. and Gray, W.G., 1979. General conservation equations for multi-phase systems, 2. Mass, momenta, energy, and entropy equations. Adv. Water Resour., 2(3): 191-203.

Hughes, T.J.R. and Brooks, A., 1982. A theoretical framework for Petrov-Galerkin methods with discontinuous weighting functions: Application of the streamline-upwind procedure. In: Finite Elements in Fluids. Wiley, New York, NY, pp. 47-65. 
Kaluarachchi, J.J. and Parker, J.C., 1990. Modeling multicomponent organic chemical transport in three-fluid-phase porous media. J. Contam. Hydrol., 5: 349-374.

Katyal, A.K., Kaluarachchi, J.J. and Parker, J.C., 1991. A two dimensional finite element program for multiphase flow and multicomponent transport. U.S. Environ. Prot. Agency, Washington, DC, CR-814320.

Lapidus, L. and Pinder, G.F., 1982. Numerical Solution of Partial Differential Equations in Science and Engineering. Wiley, New York, NY, $677 \mathrm{pp}$.

Mackay, D.M., Roberts, P.V. and Cherry, J.A., 1985. Transport of organic contaminants in groundwater. Environ. Sci. Technol., 19(5): 384-392.

Mayer, A.S. and Miller, C.T., 1990. A compositional model for simulating multiphase flow, transport and mass transfer in groundwater systems. In: G. Gambolati, et al. (Editors), Computational Methods in Subsurface Hydrology, Venice, Italy. Springer, New York, NY, pp. 217-222.

Mualem, Y., 1976. A new model for predicting the hydraulic conductivity of unsaturated porous media. Water Resour. Res., 12(3): 512-522.

Parker, J.C., Kuppusamy, T. and Lien, B.H., 1987a. Modeling immiscible organic chemical transport in soils and groundwater. In: Proceedings of the International Conference on Groundwater Contamination: Use of Models in Decision Making, International Groundwater Modeling Center, Amsterdam. Martinus Nijhoff, The Hague.

Parker, J.C., Lenhard, R.J. and Kuppusamy, T., 1987b. A parametric model for constitutive properties governing multiphase fluid conduction in porous media. Water Resour. Res., 23(4): $618-624$.

Powers, S.E., Loureiro, C.O., Abriola, L.M. and Weber, Jr., W.J., 1991. Theoretical study of the significance of non-equilbrium dissolution of non-aqueous phase liquids in subsurface systems. Water Resour. Res., 27(4): 463-478.

Powers, S.E., Abriola, L.M. and Weber, Jr., W.J., 1992. An experimental investigation of NAPL dissolution in saturated subsurface systems: Steady-state mass transfer rates. Water Resour. Res., 28(10) 2691-2707.

Reeves, H.W., 1993. Volatilization and vapor phase transport of organic contaminants in the subsurface. Ph.D. Dissertation, The University of Michigan, Ann Arbor, MI, $271 \mathrm{pp}$.

Reeves, H.W. and Abriola, L.M., 1988. A decoupled approach to the simulation of flow and transport of non-aqueous phase contaminants through porous media. In: M.A. Celia, L.A. Ferrand, C.A. Brebbia, W.G. Gray and G.F. Pinder (Editors), Computational Methods in Water Resources, Vol. 1: Modeling Surface and Sub-surface Flows, Proceedings of the VII International Conference, MIT, USA. Elsevier, Amsterdam (co-published with Computational Mechanics Publications, Southampton), pp. 147-152.

Reid, R.C., Prausnitz, J.M. and Sherwood, T.K., 1977. The Properties of Gases and Liquids. McGraw-Hill, New York, NY, 3rd ed., 688 pp.

Ryan, P.A. and Cohen, Y., 1991. One-dimensional subsurface transport of nonaqueous phase liquid containing sparingly water soluble organics: A front-tracking model. Water Resour. Res., 27(7): 1487-1500.

Sleep, B.E. and Sykes, J.F., 1990. Numerical simulation of three phase multi-dimensional flow in porous media. In: G. Gambolati, et al. (Editors), Computational Methods in Subsurface Hydrology, Venice, Italy. Springer, New York, NY, pp. 211-216.

Treybal, R.E., 1980. Mass-Transfer Operations. McGraw-Hill, New York, NY, 784 pp.

van Genuchten, M.Th., 1980. A closed-form equation for predicting the hydraulic conductivity of unsaturated soils. Soil Sci. Soc. Am., 44: 892-898. 